\title{
Apport de la Méthode de Runge Kutta d'ordre 4 dans la Dynamique de Système Mécanique
}

\author{
Contribution of the Runge Kutta order 4 method in the dynamic of
} Mechanical System

\author{
Trésor Kanyiki ${ }^{1}$ \\ ${ }^{1}$ Département d'éléctromécanique, Faculté Polytechnique, Université de Lubumbashi, République Démocratique du \\ Congo, tresorkanyiki@gmail.com
}

\begin{abstract}
RÉSUMÉ. L'objectif de cet article est de résoudre les équations différentielles de Lagrange d'un système discret. En effet, la modélisation et la simulation sont les étapes importantes dans l'analyse d'un système mécanique. La modélisation permet d'écrire les équations différentielles qui décrivent le comportement dynamique et la simulation permet d'en produire la résolution. Nous avons présenté la méthode de Runge Kutta d'ordre 4 programmé sous Matlab pour résoudre les équations différentielles d'un système discret.

ABSTRACT. The purpose of this article is to solve the differential equations of Lagrange of a discrete system. Indeed, modeling and simulation are important steps in mechanical analysis. Modeling makes it possible to write the differential equations that describe the dynamic behavior and the simulation makes it possible to produce the resolution. We presented the Runge Kutta order 4 method under Matlab to solve the differential equations of a discrete system.

MOTS-CLÉS. Système discret, équations différentielles, Runge Kutta d'ordre 4, Matlab.

KEYWORDS. Discrete system, differential equations, Runge Kutta order 4, Matlab.
\end{abstract}

\section{Introduction}

La mécanique rationnelle que l'on divise en statique, cinématique et dynamique joue un rôle important dans l'histoire des techniques et des sciences. De tout temps, l'homme essaye de comprendre les lois qui régissent le mouvement ou l'équilibre des corps qui l'entourent [PED 11]. La mécanique rationnelle repose sur des principes élémentaires, sa période de gestation qui a permis d'en établir les lois essentielles part depuis les formulations d'Aristote, quatre siècles avant Jésus-Christ, jusqu'à la formulation par Euler en 1760 des équations du mouvement d'un solide sollicité par une force quelconque [LEG 14].

On procède à une modélisation d'un phénomène lorsqu'on estime que sa compréhension est suffisante. Les étapes importantes à la maitrise et la connaissance de tout phénomène physique sont : l'observation, la compréhension, la modélisation et la simulation [REV 13]. Pour répondre à l'évolution des disciplines technique (robotique, construction ferroviaires et routiers, aéronautiques, biomécanique, ...), la dynamique des systèmes s'est développée cette dernière décennie. Ce développement est dû suite aux possibilités offertes par une informatique accessible et de plus en plus puissante. Les moyens de calcul ont fait de la mécanique rationnelle une science véritablement appliquée.

La modélisation est une troisième étape dans la solution d'un problème de la mécanique, elle a pour objectif de produire sa description mathématique. Cette description mathématique est obtenue à partir des lois de la mécanique rationnelle. Ces lois ne peuvent pas être appliquées directement sur le système réel ; il est crucial d'en introduire des hypothèses qui simplifient le problème afin que ces lois puissent être appliquées. Il s'agit de la création d'un modèle physique. On appelle modèle mathématique, l'application des lois de la mécanique rationnelle au modèle physique. 
La dynamique est une branche de la mécanique qui établit une relation mathématique entre les forces appliquées sur un corps et le mouvement qui en résulte. Pour cela, plusieurs formalismes sont développés ; la méthode de Lagrange est une méthode énergétique qui permet d'obtenir les équations différentielles qui décrivent le comportement dynamique d'un modèle physique. Dans [ELI 98], on a établi les expressions des équations de Lagrange à partir du principe des travaux virtuels de D'Alembert, de la dynamique newtonienne et des principes du calcul variationnel (théorie d'EulerLagrange). La solution des équations de Lagrange d'un système discret, donne la loi des mouvements du système. La résolution manuelle par les méthodes classiques de ces équations différentielles s'avère très ardue.

Les méthodes numériques se sont développées ces dernières années avec le développement de l'informatique, qui permet de calculer les itérations pour des systèmes d'équations complexes [CIA 96]. Nous avons programmé la méthode de Runge Kutta d'ordre 4 sur Matlab pour résoudre les équations différentielles des systèmes discrets. L'outils informatique simplifie l'écriture et le calcul des solutions approchées par la méthode de Runge Kutta d'ordre 4.

\section{Méthode de Lagrange}

Il n'existe pas dans la nature les éléments sans masses ni rigidité. Donc chaque particule d'un élément a un mouvement indépendant. Ceci implique que pour décrire la position de tous les points par rapport à un référentiel inertiel, il faut une infinité de degré de liberté. Cependant, en vibration, on peut formuler les hypothèses permettant de réduire le nombre de degré de liberté avec une certaine précision. Cela se fait en décrivant le système par un nombre limité de corps rigides reliés par des amortissements sans masse et des raideurs (figure 1). Cette procédure est appelée discrétisation, elle conduit à un système à plusieurs degré liberté [PED 88].

Soit un système de $\mathrm{N}$ particules ponctuelles, $m_{j}$ est la masse inerte de la particule $\mathrm{n}^{\circ} \mathrm{j}$. La dynamique du système des particules est décrite par l'application du principe de d'Alembert (la force appliquée sur une particule est égale à la variation de sa quantité de mouvement par rapport au temps). Chaque particule est soumise à une force $F_{j}$ et est animée d'une vitesse $V_{j}$ dans un référentiel orthonormé $(O, \vec{i}, \vec{j}, \vec{k})$. Sous l'effet de la force $F_{j}$, chaque particule acquiert une accélération $a_{j}=\frac{d V_{j}}{d t}$. La force $F_{j}$ exercée sur une particule $M_{j}$ est la somme des forces d'interaction entre les autres particules et les forces extérieures $F_{E, j}$ [ELI 11].

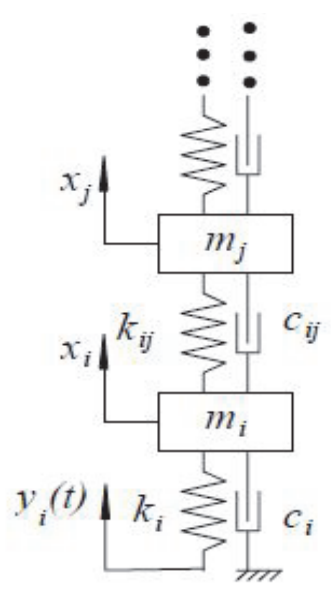

Figure 1. Système discret à $N$ degré de liberté. 


\subsection{Modélisation mathématique}

Les équations de mouvement d'un système vibratoire sont obtenues en utilisant les équations de Lagrange :

$$
-\frac{d}{d t}\left(\frac{\partial T}{\partial \dot{q}_{i}}\right)+\frac{\partial T}{\partial q_{i}}-\frac{\partial V}{\partial q_{i}}-\frac{\partial F}{\partial \dot{q}_{i}}+Q_{j}^{(n)}=0
$$

Dans l'équation (1), T est la fonction énergie cinétique du système, $q_{j}=\frac{\partial q_{j}}{\partial t}$ la vitesse généralisée, $Q_{j}^{(n)}$ la force généralisée non-conservative correspondant à la coordonnée généralisée $q_{j}$ et $\mathrm{V}$ la fonction énergie potentielle du système.

Les forces généralisées $Q_{j}^{(n)}$ peuvent être des forces externes qui ne dérivent pas d'un potentiel ou dissipatif (amortissement). Considérons une masse $m_{k}$ et les forces $F_{x k}, F_{y k}$ et $F_{z k}$ qui lui sont appliquées. La force généralisée est :

$$
Q_{j}^{(n)}=\sum_{k}\left(F_{x k} \frac{\partial x_{k}}{\partial q_{j}}+F_{y k} \frac{\partial y_{k}}{\partial q_{j}}+F_{z k} \frac{\partial z_{k}}{\partial q_{j}}\right)
$$

Lorsque l'équation de Lagrange est appliquée sur un système discret, elle prend la forme suivante :

$$
[M]\{\overrightarrow{\ddot{q}}\}+[C]\{\overrightarrow{\dot{q}}\}+[K]\{\vec{q}\}=\{F\}
$$

Avec

$[M]=\left[\begin{array}{ccccc}m_{11} & m_{12} & m_{13} & \cdots & m_{1 n} \\ m_{21} & m_{22} & m_{23} & \cdots & m_{2 n} \\ \vdots & & & & \\ m_{n 1} & m_{n 2} & m_{n 3} & \cdots & m_{n n}\end{array}\right]$

$[C]=\left[\begin{array}{ccccc}c_{11} & c_{12} & c_{13} & \cdots & c_{1 n} \\ c_{21} & c_{22} & c_{23} & \cdots & c_{2 n} \\ \vdots & & & & \\ c_{n 1} & c_{n 2} & c_{n 3} & \cdots & c_{n n}\end{array}\right]$

$[K]=\left[\begin{array}{ccccc}k_{11} & k_{12} & k_{13} & \cdots & k_{1 n} \\ k_{21} & k_{22} & k_{23} & \cdots & k_{2 n} \\ \vdots & & & & \\ k_{n 1} & k_{n 2} & k_{n 3} & \cdots & k_{n n}\end{array}\right]$

- Le système est dit couplé par les masses ou par les inerties si la matrice masse n'est pas diagonale ;

- Le système est dit couplé par les raideurs ou élastiquement si la matrice de raideur n'est pas diagonale ;

- Le système est couplé par les vitessements ou par les amortissements si la matrice d'amortissement n'est pas diagonal ;

- Les couplages par raideur est dit statique et les couplages par amortissement et par masses sont dits couplages dynamiques. 


\subsubsection{Modèle physique}

Considérons un corps 1 qui est suspend à une barre 2 (figure 2). Déplaçons le corps 1 de sa position d'équilibre avant de le relâcher, chaque point du système aura un mouvement oscillatoire indépendant. Un nombre infini de coordonnées $x_{i}$ peut décrire le mouvement du système [PED 98].

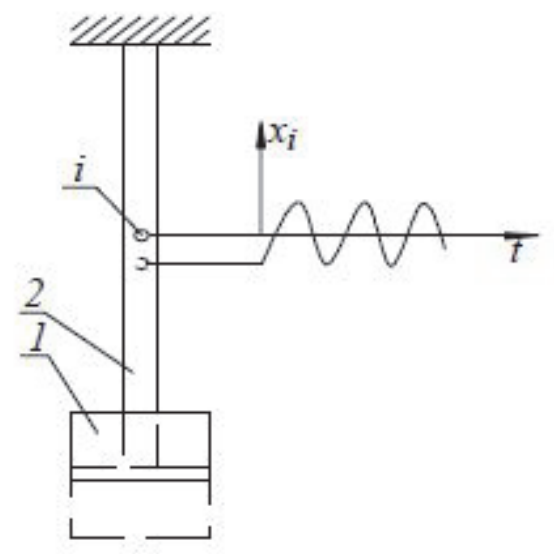

Figure 2. Système physique simple

Nous voyons qu'un système réel possède une infinité de degré de liberté. Les hypothèses permettent de réduire le nombre degré de liberté. Pour le cas de la figure 2, on peut faire les hypothèses suivantes : le corps 1 est rigide et la barre 2 n'a pas de masse. Une coordonnée seulement suffit pour décrire le mouvement du système. Le déplacement $x$ du corps rigide 1 est choisi comme coordonnée indépendante.

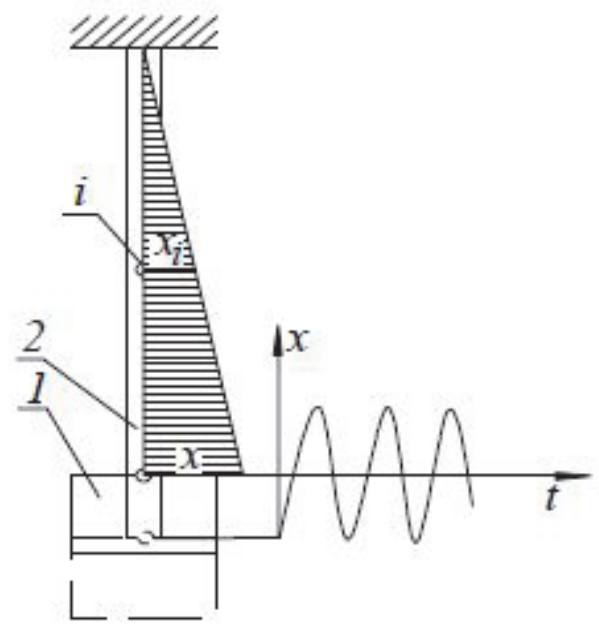

Figure 3. Système à un degré de liberté

La position $x_{i}$ de chaque point de notre système dépend de $x$. La position instantanée en fonction de $x$ est montrée à la figure 3 pour une barre uniforme. La figure 4 illustre le modèle discrétisé du problème de la figure 2 . Il s'agit du système à un degré de liberté idéalisé par un système masseressort-amortissement. 


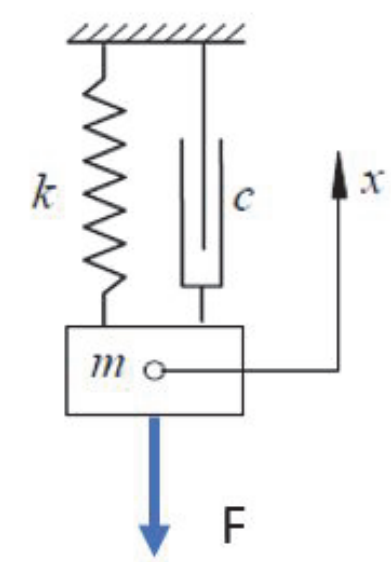

Figure 4. Modèle d'un système à un degré de liberté

Les équations différentielles qui décrivent le comportement dynamique du système discrétisé sont difficiles à résoudre manuellement, surtout lorsque le nombre de degré de liberté augmente. Cet article présente la méthode numérique de Runge Kutta d'ordre 4 pour résoudre ces équations différentielles. Nous avons développé un algorithme sous Matlab basé sur la méthode de Runge Kutta d'ordre 4 afin de résoudre les équations différentielles de Lagrange. Leurs résolutions numériques manuelles par la méthode de Runge Kutta d'ordre 4 est ardue.

\section{Méthode numérique de Runge Kutta d'ordre 4}

La méthode de Runge Kutta d'ordre 4 permet de résoudre les équations différentielles de la forme :

$$
\left\{\begin{array}{c}
y^{\prime}(x)=f(x, y(x)) \\
y(0)=y_{0}
\end{array}\right.
$$

La solution de cette équation est de la forme :

$$
y_{n+1}=y_{n}+\frac{1}{6}\left(k_{1}+2 k_{2}+2 k_{3}+k_{4}\right)
$$

avec

$$
\begin{aligned}
& k_{1}=h f\left(x_{n}, y_{n}\right) \\
& k_{2}=h f\left(x_{n}+\frac{h}{2}, y_{n}+\frac{k_{1}}{2}\right) \\
& k_{3}=h f\left(x_{n}+\frac{h}{2}, y_{n}+\frac{k_{2}}{2}\right) \\
& k_{4}=h f\left(x_{n}+h, y_{n}+k_{3}\right)
\end{aligned}
$$

En approximant une fonction par la méthode de Runge Kutta d'ordre 4, on comment l'erreur de l'ordre $h^{4}$.

\section{Résolution des problèmes}

Voyons maintenant comment résoudre les problèmes discrétisés de la dynamique de Lagrange à l'aide de la méthode de Runge Kutta d'ordre 4. 


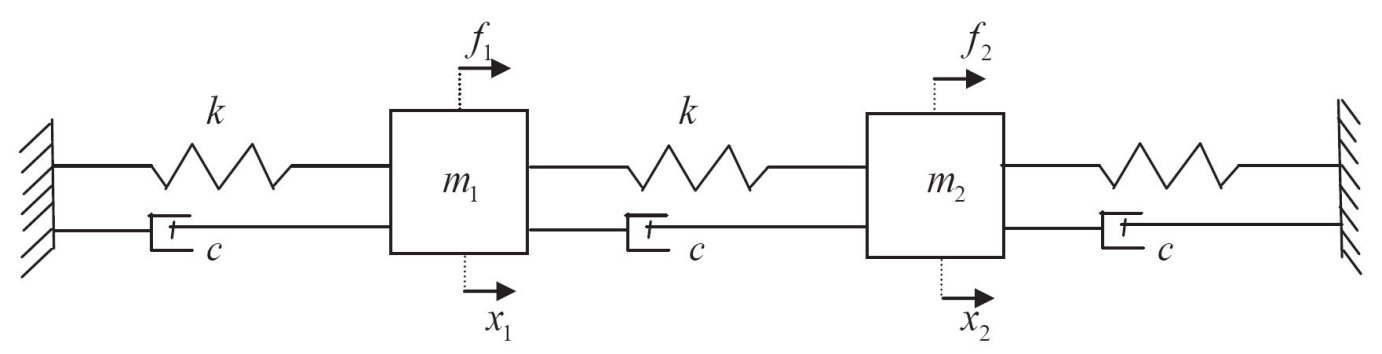

Figure 5. Modèle d'un système à deux degré de liberté

Considérons un système masses amorti à deux degrés de liberté (figure 5). L'énergie cinétique, l'énergie potentielle et les forces dissipatives de ce système sont par :

$$
\begin{aligned}
& T=\frac{1}{2} m_{1} \dot{q}_{1}^{2}+\frac{1}{2} m_{2} \dot{q}_{2}^{2} \\
& V=\frac{1}{2} k q_{1}^{2}+\frac{1}{2} k\left(q_{2}+q_{1}\right)^{2}+\frac{1}{2} k q_{2}^{2} \\
& F=\frac{1}{2} k \dot{q}_{1}^{2}+\frac{1}{2} k\left(\dot{q}_{2}+\dot{q}_{1}\right)^{2}+\frac{1}{2} k \dot{q}_{2}^{2} \\
& Q_{1}=f_{1} \text { et } Q_{2}=f_{2}
\end{aligned}
$$

En appliquant l'équation de Lagrange (1), aux relations (7), (8), (9) et (10); nous obtenons le système d'équation différentielle suivant:

$$
\left[\begin{array}{cc}
m_{1} & 0 \\
0 & m_{2}
\end{array}\right]\left\{\begin{array}{l}
\ddot{q}_{1} \\
\ddot{q}_{2}
\end{array}\right\}+\left[\begin{array}{cc}
2 c & -c \\
-c & 2 c
\end{array}\right]\left\{\begin{array}{l}
\dot{q}_{1} \\
\dot{q}_{2}
\end{array}\right\}+\left[\begin{array}{cc}
2 c & -c \\
-c & 2 c
\end{array}\right]\left\{\begin{array}{l}
q_{1} \\
q_{2}
\end{array}\right\}=\left\{\begin{array}{l}
f_{1} \\
f_{2}
\end{array}\right\}
$$

La resolution de ce système d'équations, donne les lois des mouvements de masse $m_{1}$ et $m_{2}$. Pour utiliser la méthode de Runge Kutta d'ordre 4, nous procédons de la manière suivante :

$\dot{q}_{1}=q_{3}$

$\dot{q}_{2}=q_{4}$

$\dot{q}_{3}=\frac{f_{1}-2 c q_{3}+c q_{4}-2 k q_{1}+k q_{2}}{m_{1}}$

$\dot{q}_{4}=\frac{f_{2}+c q_{3}-2 c q_{4}+k q_{1}-2 k q_{2}}{m_{2}}$

Ces relations sous forme matricielle deviennent :

$$
\left\{\begin{array}{l}
\dot{q}_{1} \\
\dot{q}_{2} \\
\dot{q}_{3} \\
\dot{q}_{4}
\end{array}\right\}=\left[\begin{array}{cccc}
0 & 0 & 1 & 0 \\
0 & 0 & 0 & 1 \\
-\frac{2 k}{m_{1}} & \frac{k}{m_{1}} & \frac{-2 c}{m_{1}} & \frac{c}{m_{1}} \\
\frac{k}{m_{2}} & -\frac{2 k}{m_{2}} & \frac{c}{m_{2}} & \frac{-2 c}{m_{2}}
\end{array}\right]\left\{\begin{array}{l}
q_{1} \\
q_{2} \\
q_{3} \\
q_{4}
\end{array}\right\}+\left\{\begin{array}{c}
0 \\
0 \\
\frac{f_{1}}{m_{1}} \\
\frac{f_{2}}{m_{2}}
\end{array}\right\}
$$

A partir des relations (5), (6) et (12), nous avons écrit un code de calcul sous Matlab, ce code a permis de tracer les figures de 6 à 12 selon le contexte de l'analyse dynamique.

Application numérique

$\mathrm{m}_{1}=0.5 \mathrm{~kg}, \mathrm{~m}_{2}=2 \mathrm{~kg}, \mathrm{k}=5 \mathrm{~N} / \mathrm{m}$ et $\mathrm{c}=2 \mathrm{~N} / \mathrm{m}$ 
On déplace la masse $m_{1}$ vers la droite en appliquant une force $f_{1}=0.5 \mathrm{~N}$ et la masse $\mathrm{m}_{2}$ vers la droite en appliquant une force $\mathrm{f}_{2}=0.9 \mathrm{~N}$. Le résultat se trouve à la figure 6 .

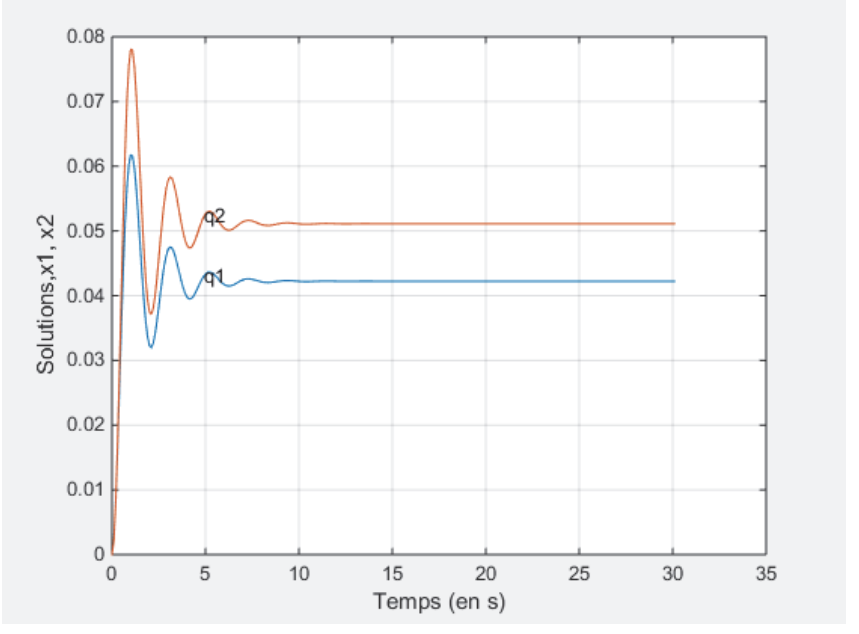

Figure 6. Simulation sous Matlab de la méthode de Runge Kutta d'orde 4

Sur figure 6, le déplacement des masses s'arrête $7 \mathrm{sec}$ après avoir appliquée les forces constantes sur ces masses. L'amplitude maximale de $\mathrm{m}_{2}$ est d'environ $0.08 \mathrm{~m}$ et celle de $\mathrm{m}_{1}$ est d'environ $0.06 \mathrm{~m}$. A présent déplaçons la masse $m_{1}$ à gauche et la masse $m_{2}$ à droite, en gardant la même intensité des forces.

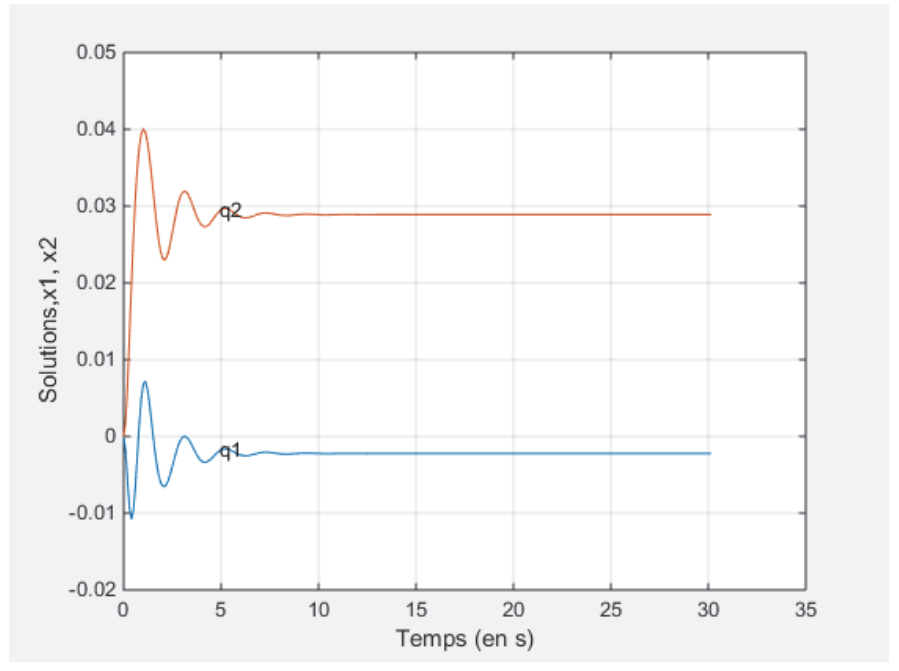

Figure 7. Simulation sous Matlab de la méthode de Runge Kutta d'orde 4

Cette fois-ci, nous constatons sur la figure 7 que la masse $m_{2}$ atteint une amplitude maximale de $0.04 \mathrm{~m}$ et la masse $\mathrm{m}_{1}$ atteint un déplacement maximal vers la gauche de $0.01 \mathrm{~m}$. Le déplacement de deux masses s'arrête $7 \mathrm{sec}$ après l'application des forces. Maintenant gardons les mêmes intensités de force, déplaçons les masses vers la droite en considérant que l'amortissement est nul, c=0. 


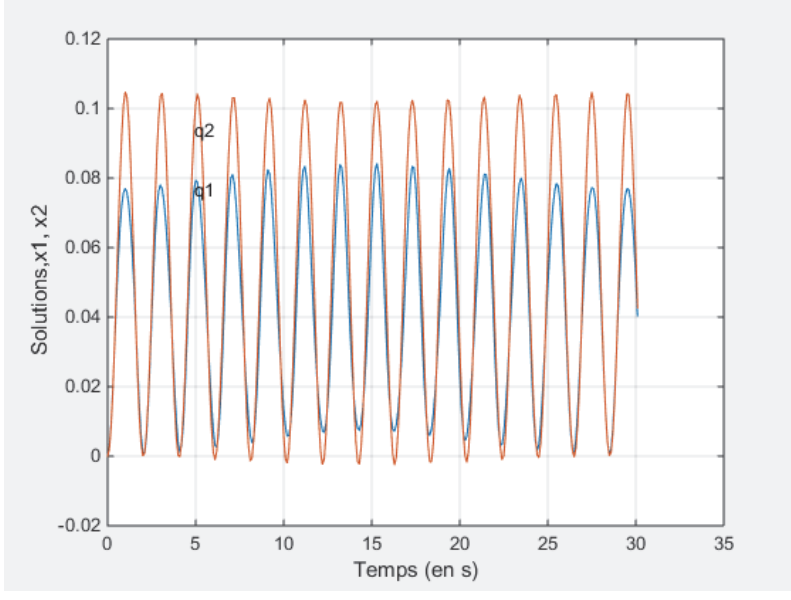

Figure 8. Simulation sous Matlab de la méthode de Runge Kutta d'orde 4

Sur la figure 8, nous voyons un mouvement sinusoïdale de même fréquence pour les deux masses, elles ont chacune un déplacement maximal de $0.1 \mathrm{~m}$ pour $\mathrm{m}_{2}$ et $0.08 \mathrm{~m}$ pour $\mathrm{m}_{1}$. Considérons un amortissement exponentiel appliqué au système, $\mathrm{c}=\exp (-0.1 \mathrm{t})$.

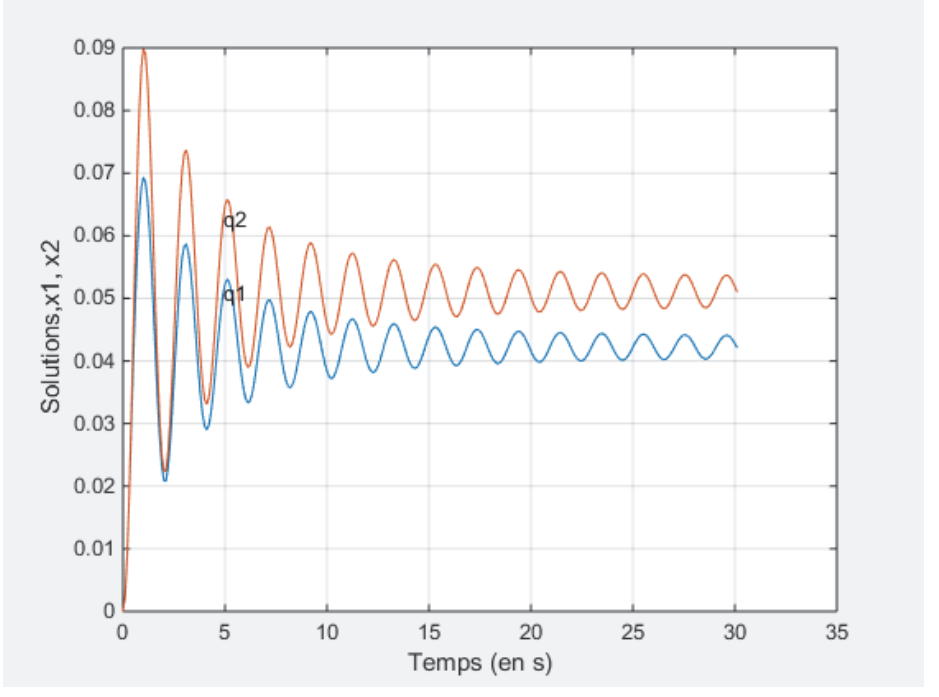

Figure 9. Simulation sous Matlab de la méthode de Runge Kutta d'orde 4

Nous voyons sur la figure 9 , que les deux masses $m_{1}$ et $m_{2}$ atteignent un déplacement maximal respectivement de $0.07 \mathrm{~m}$ et $0.09 \mathrm{~m}$; les deux mouvements deviennent sinusoïdal après 15 sec. Considérons que les deux masses sont sollicitées par deux forces sinusoïdales de mêmes fréquences $\mathrm{f}_{1}=0.5 * \sin (0.1 * \mathrm{t}), \mathrm{f}_{2}=0.9 * \sin (0.1 * \mathrm{t})$ et $\mathrm{c}=2 \mathrm{~N} / \mathrm{m}$. 


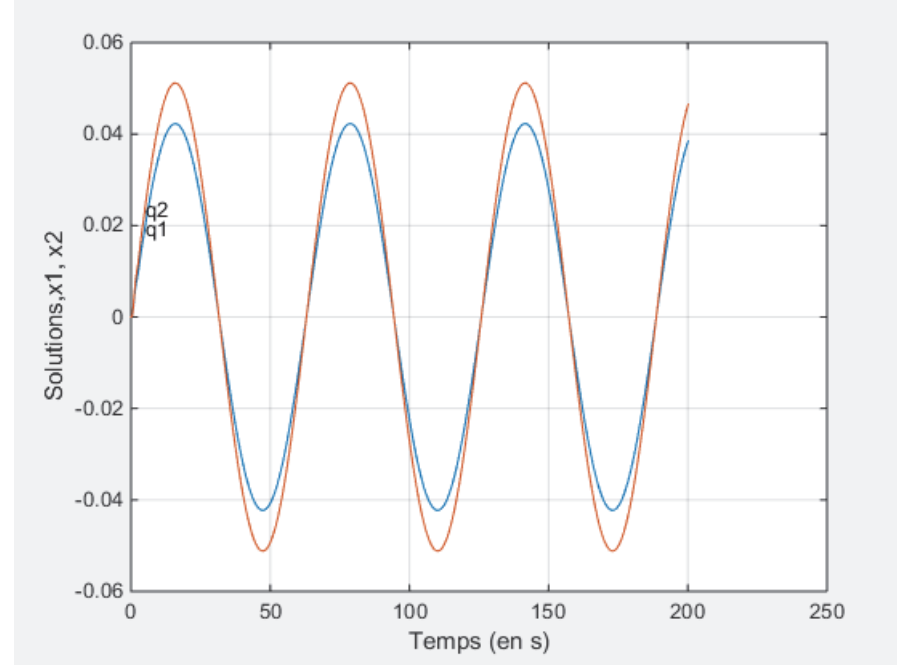

Figure 10. Simulation sous Matlab de la méthode de Runge Kutta d'orde 4

Sur la figure 10, les deux masses ont un mouvement sinusoïdal de même fréquence et d'amplitudes de déplacement différentes. Maintenant prenons la force $\mathrm{f}_{1}=0.5 * \sin (0.1 * t), \mathrm{f}_{2}=-0.9 * \sin (0.1 * \mathrm{t})$ et $\mathrm{c}=2 \mathrm{~N} / \mathrm{m}$; les deux masses auront toujours la même fréquence, la masse $\mathrm{m}_{1}$ a une amplitude faible par rapport à la masse $\mathrm{m}_{2}$.

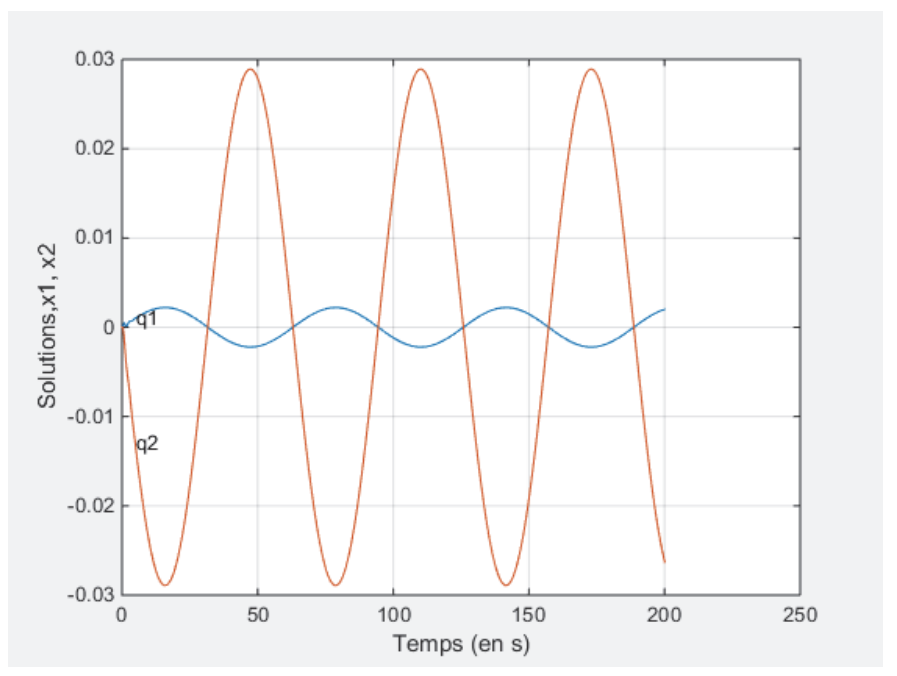

Figure 11. Simulation sous Matlab de la méthode de Runge Kutta d'orde 4

Prenons : $\mathrm{f}_{1}=0.5 * \sin (0.1 * \mathrm{t}), \mathrm{f}_{2}=0.9 * \sin (0.1 * \mathrm{t})$ et $\mathrm{c}=\exp (-0.1 \mathrm{t})$, nous voyons que les deux masses (figure 11) ont un mouvement sinusoïdal d'amplitude différente. 


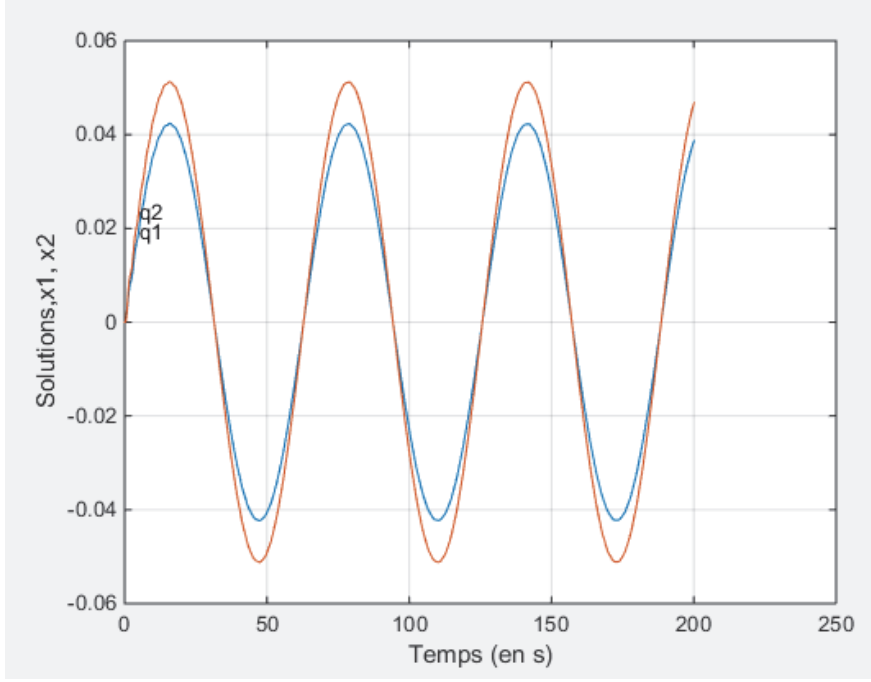

Figure 12. Simulation sous Matlab de la méthode de Runge Kutta d'orde 4

Prenons un deuxième exemple ; sur la figure 13, nous avons un système à trois degrés de liberté, ce système mécanique est sollicité par une force d'excitation au niveau de la masse $\mathrm{m}_{3}$.

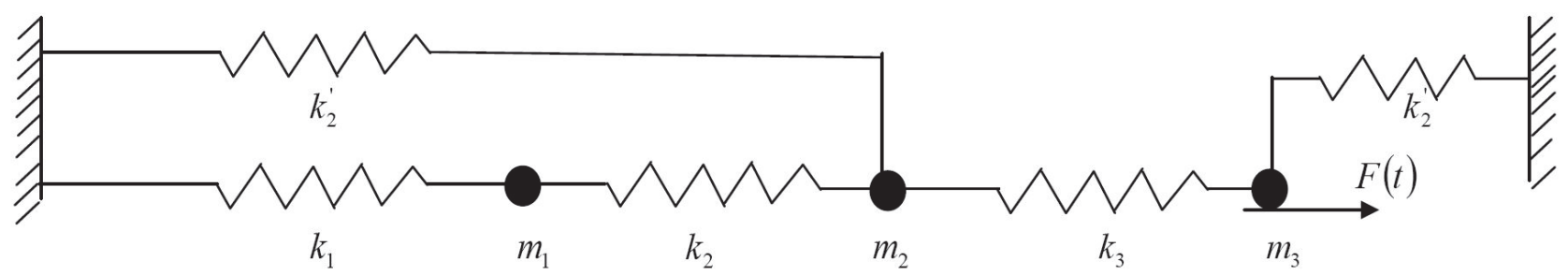

Figure 13. Simulation sous Matlab de la méthode de Runge Kutta d'orde 4

En appliquant les équations de Lagrange (1) sur le système de la figure 13, en passant par les énergies cinétiques et potentielles du système, nous obtenons le système d'équations (13).

$$
\left[\begin{array}{ccc}
m_{1} & 0 & 0 \\
0 & m_{2} & 0 \\
0 & 0 & m_{3}
\end{array}\right]\left\{\begin{array}{l}
\ddot{q}_{1} \\
\ddot{q}_{2} \\
\ddot{q}_{3}
\end{array}\right\}+\left[\begin{array}{ccc}
k_{1}+k_{2} & -k_{2} & 0 \\
-k_{2} & k_{2}+k_{3}+k_{2}^{\prime} & -k_{3} \\
0 & -k_{3} & k_{3}+k_{3}^{\prime}
\end{array}\right]\left\{\begin{array}{l}
q_{1} \\
q_{2} \\
q_{3}
\end{array}\right\}=\left\{\begin{array}{c}
0 \\
0 \\
F(t)
\end{array}\right\}
$$

Afin d'utiliser la méthode de Runge Kutta pour résoudre ce système d'équations (13), nous posons $\dot{q}_{1}=q_{5}, \dot{q}_{2}=q_{6}$ et $\dot{q}_{3}=q_{6}$, le système d'équations (13), devient :

$$
\left\{\begin{array}{l}
\dot{q}_{1} \\
\dot{q}_{2} \\
\dot{q}_{3} \\
\dot{q}_{4} \\
\dot{q}_{5} \\
\dot{q}_{6}
\end{array}\right\}=\left[\begin{array}{cccccc}
0 & 0 & 0 & 1 & 0 & 0 \\
0 & 0 & 0 & 0 & 1 & 0 \\
0 & 0 & 0 & 0 & 0 & 1 \\
-\frac{\left(k_{1}+k_{2}\right)}{m_{1}} & \frac{k_{2}}{m_{1}} & 0 & 0 & 0 & 0 \\
\frac{k_{2}}{m_{2}} & -\frac{\left(k_{1}+k_{4}+k_{2}^{\prime}\right)}{m_{2}} & \frac{k_{3}}{m_{2}} & 0 & 0 & 0 \\
0 & \frac{k_{3}}{m_{3}} & -\frac{\left(k_{3}+k_{3}^{\prime}\right)}{m_{3}} & 0 & 0 & 0
\end{array}\right\}\left\{\begin{array}{l}
q_{1} \\
q_{2} \\
q_{3} \\
q_{4} \\
q_{5} \\
q_{6}
\end{array}\right\}+\left\{\begin{array}{c}
0 \\
0 \\
0 \\
0 \\
0 \\
F(t) \\
m_{3}
\end{array}\right\}
$$


Les relations (5), (6) et (14) sont programmées sous Matlab; les valeurs numériques suivantes ont été utilisées dans le code Matlab : $\mathrm{m}_{1}=\mathrm{m}_{3}=0.5 \mathrm{~kg}, \mathrm{~m}_{2}=1, \mathrm{k}_{1}=\mathrm{k}_{2}=\mathrm{k}_{3}=\mathrm{k}_{3}{ }_{3}=1, \mathrm{k}_{2}{ }_{2}=2$ et $\mathrm{f}_{1}=0.5 \mathrm{~N}$. La figure 14, donne les amplitudes de déplacement des masses, il est claire que sous l'action de la force $\mathrm{f}_{1}$, les trois masses oscillent de manière aléatoire autour de la position d'équilibre.

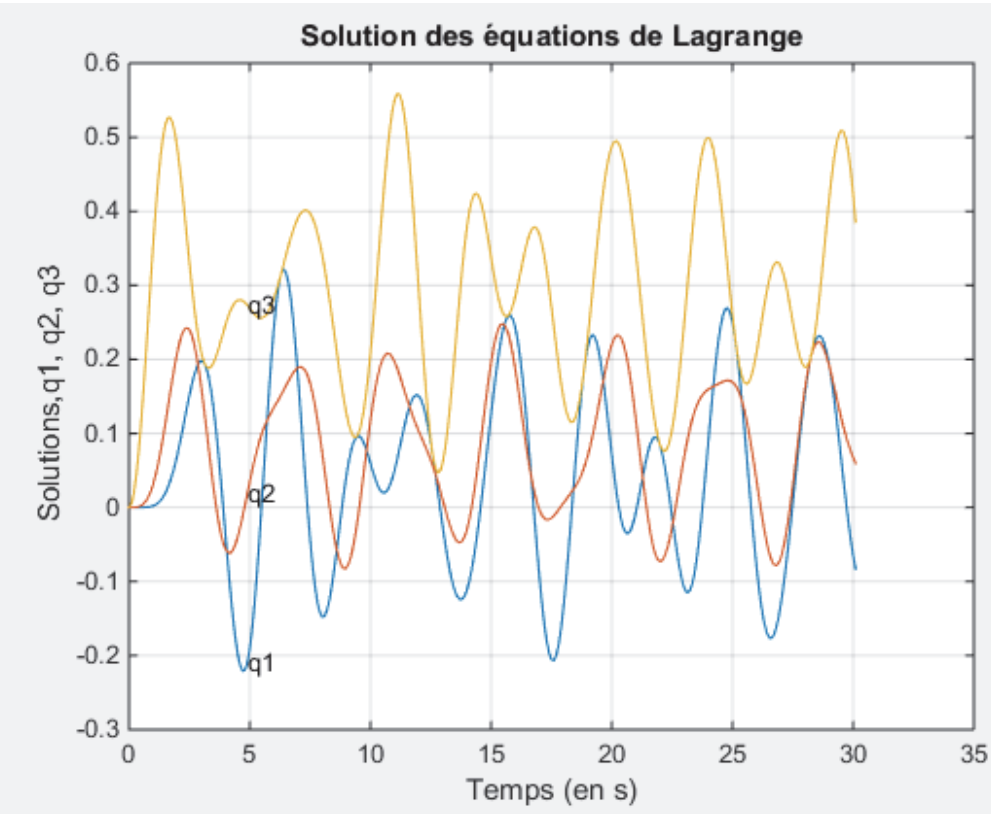

Figure 14. Simulation sous Matlab de la méthode de Runge Kutta d'orde 4

En appliquant une force sinusoïdale $(\mathrm{F}=0.5 * \sin (0.5 * \mathrm{t}))$ sur la masse $\mathrm{m}_{3}$, les résultats sont des fonctions aléatoires qui sont représentées à la figure 15 .

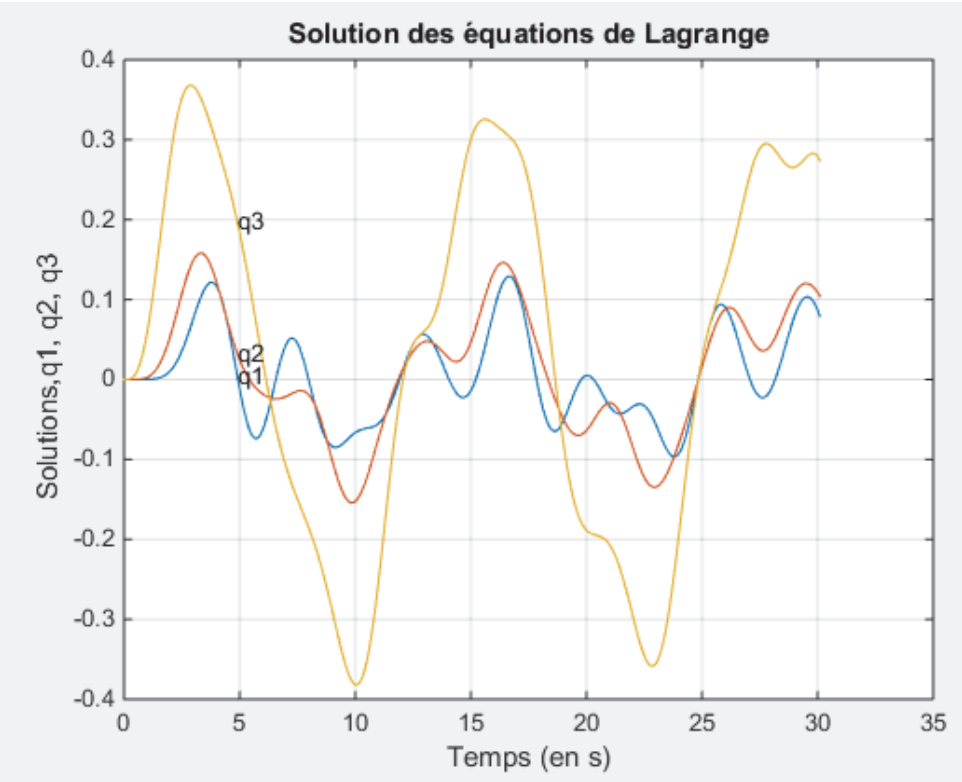

Figure 15. Simulation sous Matlab de la méthode de Runge Kutta d'orde 4

\section{Conclusion}

Dans ce travail, nous avons effectué l'analyse dynamique d'un système discret. Il est clair que l'approche de Lagrange conduit aux équations différentielles dont la résolution analytique est ardue. L'approche numérique est difficile manuellement, à partir de deux exemples de système discret ; nous 
avons effectué l'analyse dynamique à l'aide de la méthode numérique de Runge Kutta d'ordre 4, différent cas ont été abordé lors de cette analyse. La programmation sous Matlab de la méthode de Runge Kutta d'ordre 4 pour résoudre les équations différentielles qui décrivent le comportement dynamique des systèmes discret est un excellant outil pour l'analyse des systèmes discret à plusieurs degré de liberté ; il permet de connaitre l'évolution des déplacements de masse uniquement. De ce fait, le temps de calcul sur ordinateur augmente ; mais cet outil ne permet pas d'effectuer l'analyse modale d'un système discret. L'analyse modale permet de déterminer les caractéristiques modales d'un système discret.

La méthode de Runge Kutta d'ordre 4 permet de résoudre les équations différentielles de la dynamique de Lagrange à coefficient constant. Il impossible d'utiliser cette méthode lorsque les coefficients des équations différentielles sont variables, et dépendent des paramètres de configuration.

\section{Bibliographie}

[CIA 96] CIARLET P.G., Introduction à l'analyse numérique matricielle et à l'optimisation, Masson, 1990.

[PRE 13] PREUMONT A., Twelve lectures on structural dynamics, Université libre de Bruxelles, 2013.

[PRE 02] PREUMONT A., Vibration control of active structures: an introduction, $2^{\text {nd }}$ Edition Kluwer academic publishers, 2002.

[REV 13] REVEILLON J., cours de simulation et modélisation numérique, Université de Roueu, 2013.

[PED 88] Pedro M.D., PAHUd P., Mécanique vibratoire: Système discrets linéaire, Presse polytechnique Romandes, 1988 .

[ELI 11] ELIE F., Introduction aux équations de Lagrange en mécanique analytique, Accueil, 2011.

[VER 03] VerLInden O., Computer Aided Analysis of multibody systems, Faculté polytechnique de Mons, 2003.

[KOP 12] KOPPER C., Principes variationnels et mécanique analytique, Enseignement diversifié 1, 2012.

[MOH 03] Mohammadi B., SAIAC J.H., Pratique de la simulation numérique, Dunod, 2003.

[POT 09] Potel C., Gatignol P., Cours de mécanique-vibration, université du Maine-UFR Sciences et techniques, 2009.

[GON 05] GONCALVES E., Résolution numérique, discrétisation des EDP et EDO, Institut national polytechnique de Grenoble, 2005.

[BEU 09] BEUNEU J., Cours d'algorithmes pour le calcul scientifique, Ecole universitaire d'ingénieure de Lille, 2009.

[LEG 14] LEGOT V., Cours de mathématique et méthodes numériques, Université catholique de Louvain, 2014.

[THE 86] THEODOR R., LASCAUX P., Analyse numérique matricielle appliquée à l'art de l'ingénieur, Tome I, Masson, 1986. 